\title{
Aplicación de las metodologías EPA, mexicano e IPCC para la estimación de biogás, caso de estudio relleno sanitario Doña Juana, Bogotá - Colombia
}

\author{
Application of methodologies EPA, Mexican and IPCC for estimating biogas, a case study of the sanitary landfill \\ Doña Juana, Bogota - Colombia
}

Elizarova Urrego-Martínez1, Juan Pablo Rodríguez-Miranda²

1 Química Industrial. Msc. En Desarrollo Sustentable y Gestión Ambiental. Profesional Especializado, Secretaría Distrital de Desarrollo Económico. Bogotá, Colombia. e-mail: elizaurrego@hotmail.com

2 Ingeniero Sanitario y Ambiental. Magister en Ingeniería Ambiental. PhD (C). Profesor Asociado. Universidad Distrital Francisco José de Caldas. Director del grupo de investigación AQUAFORMAT. Bogotá, Colombia e-mail: jprodriguezm@udistrital.edu.co.

Fecha de recepción: Mayo 4 - 2015

Fecha de aceptación: Junio 28 - 2016

Urrego-Martínez E, Rodríguez-Miranda JP. Aplicación de las metodologías EPA, mexicano e IPCC para la estimación de biogás, caso de estudio relleno sanitario doña Juana, Bogotá-Colombia. Univ. Salud. 2016;18(2):338-344. DOI: http://dx.doi.org/10.22267/rus.161802.43

\begin{abstract}
Resumen
Objetivo: Realizar la comparación de las metodologías mexicano, EPA e IPCC para la estimación de biogás en un horizonte de predicción de 20 años, aplicado en un caso de estudio, en el relleno sanitario doña Juana, Bogotá, Colombia. Materiales y métodos: Estudio descriptivo para el desarrollo de la predicción de las metodologías, examinando la información secundaria obtenida de los datos iniciales de producción de Biogás en el relleno sanitario, dentro de un fenómeno homogéneo. Resultados: La producción promedio de biogás ( $\mathrm{m}^{3}$ /año) para los métodos de mexicano, EPA e IPCC, es de 2.48E+05, 2.14E+05 y 6.71E+05, respectivamente. El método con menor desviación estándar y error cuadrático medio es el mexicano. Conclusiones: La aplicación de los modelos de la EPA, Mexicano e IPCC en la estimación del biogás en un relleno sanitario, considera que la tendencia de los dos primeros métodos expuestos, es exponencial decreciente, mientras que el IPPC presenta tendencia exponencial creciente, evaluados en un periodo de predicción de 20 años. Con la determinación de la cantidad de biogás por las diferentes metodologías, se reduce la posibilidad de exposición del riesgo de largo plazo de efectos adversos en la salud pública de la comunidad adyacente a los rellenos sanitarios.
\end{abstract}

Palabras clave: Rellenos sanitarios, biogás, salud pública, metodologías, residuos sólidos urbanos. (Fuente: DeCS, Bireme).

\begin{abstract}
Objective: Compare the Mexican, EPA and IPCC methodologies for estimating biogas in a prediction horizon of 20 years applied in a case study in the landfill Doña Juana in Bogotá, Colombia. Materials and methods: A descriptive study for the development of prediction methodologies was carried out by examining the secondary information obtained from the initial data biogas production in the landfill, within a homogeneous phenomenon. Results: The average production of biogas $\left(\mathrm{m}^{3} /\right.$ year) for Mexican, EPA and IPCC methods is $2.48 \mathrm{E}+05,2.14 \mathrm{e}+05$ and $6.71 \mathrm{E}+05$, respectively. The method with lower standard deviation and root mean square error is the Mexican. Conclusions: The application of the EPA, Mexican and IPCC models in the estimation of biogas in a landfill, believes that the trend of the first two exposed methods exhibit is decreasing exponentially, while the IPPC has exponentially growing trend,
\end{abstract}


evaluated for a prediction period of 20 years. With the determination of the amount of biogas by different methodologies, the possibility of exposure of risk in the long term with adverse effects on public health of the adjacent community landfills is reduced.

Keywords: Sanitary landfill, biogas, public health, methodologies, municipal solid waste. (Source: DeCS, Bireme).

\section{Introducción}

El biogás es una mezcla de metano $\left(\mathrm{CH}_{4}\right)$ de 40 a $70 \%$, dióxido de carbono $\left(\mathrm{CO}_{2}\right)$ y otros gases (hidrogeno, sulfuros, trazas de otros gases) generado por la acción de los microrganismos especializados para descomponer la materia orgánica dentro del relleno sanitario en un entorno sin presencia de oxigeno ${ }^{1,2}$. En la actualidad, los vertederos o rellenos sanitarios son generadores de biogás que es el resultado del proceso de descomposición de los residuos sólidos especialmente aquellos que son de origen orgánico, el cual se encuentra compuesto por gas metano $\left(\mathrm{CH}_{4}\right)$, dióxido de carbono $\left(\mathrm{CO}_{2}\right)$ y trazas de compuestos orgánicos volátiles en donde, el metano es el mayor contribuyente al efecto invernadero ${ }^{3}$. La cantidad de biogás producido en los sitios de disposición final, es función de la cantidad de residuos, el tipo y edad, contenido de humedad del vertedero, la temperatura y las prácticas de manejo de éste 4 .

Es por ello que algunas de estas características son utilizadas para llevar a cabo el control adecuado de la calidad del ambiente y para esto es necesario realizar la estimación de gases de efecto invernadero en especial el biogás o gas metano, debido a que éste es variable en el tiempo y a las condiciones climáticas que se presentan en un relleno sanitario ${ }^{5} \mathrm{y}$ de manera general, aunque no se conocen las reacciones químicas que describen el proceso de digestión anaerobia en función de parámetros establecidos como temperatura, humedad, cantidad de residuos ${ }^{2}$ en la práctica, en condiciones estacionarias, la estimación de biogás generado por los residuos sólidos que se encuentran en los vertederos se puede realizar mediante metodologías como la EPA, mexicana e IPCC las cuales, son modelos empíricos que son regidos por la cinética de degradación de primer orden para la materia orgánica ${ }^{6}$. En las metodologías, se supone que la generación de biogás llega a su máximo después de un periodo de tiempo antes de que haya generación de metano, este período es de un año después de la colocación de los residuos sólidos hasta la generación de biogás; además, después de un año de disposición de los residuos sólidos urbanos (RSU), la generación de biogás disminuye de manera exponencial mientras se va consumiendo la fracción orgánica de los residuos ${ }^{7}$. De acuerdo con lo anterior, este artículo considera la aplicación de las metodologías EPA, mexicano e IPCC para la estimación de biogás, y luego la comparación de los resultados de la estimación en un horizonte de predicción de 20 años, en un caso de estudio, en el relleno sanitario doña Juana, Bogotá, Colombia.

\section{Materiales y métodos}

\section{Tipo de estudio}

El tipo de estudio fue descriptivo examinando la información secundaria obtenida de los datos de iniciales de producción de biogás en el relleno sanitario, dentro de un fenómeno homogéneo ${ }^{8}$. Las metodologías (EPA, mexicano e IPCC) fueron aplicadas en la estimación de biogás en el Relleno Sanitario Doña Juana zona II área 3 ubicado en Bogotá, Colombia, con la información secundaria tomada de los registros de biogás del Relleno Sanitario mencionado anteriormente, para el periodo comprendido entre los años 2008 y 2009 , en condiciones del funcionamiento y clausura de la celda; los datos de precipitación en el área de estudio, fueron recolectados en la estación climatológica cercana del IDEAM.

\section{Modelo EPA}

Para utilizar este modelo es necesario conocer la tasa promedio anual de residuos dispuestos, el número de años que ha estado o estuvo abierto el relleno, el número de años que el relleno ha estado cerrado, el potencial de los residuos 
dispuestos, para generar metano y la tasa de generación de metano de los residuos dispuestos ${ }^{9}$.

El estimativo de metano generado según el modelo de caída de primer orden (EPA) se expresa así: $L F G=2 \times L_{O} \times R \times\left(e^{-k \times C}-e^{-k \times T}\right)$, donde LFG es la cantidad total de gas de relleno (landfill gas) generado en el año actual o en consideración ( pies $^{3}$ ); $\mathrm{L}_{o}$ es el Potencial total de generación de metano de los residuos ( pies $^{3} /$ libra); R es el Promedio anual de residuos dispuestos durante la vida activa (libras); k es la tasa anual de generación de metano (1/año ); T es el tiempo desde la apertura del relleno (años); C es el tiempo desde la clausura del relleno (años) ${ }^{9}$.

Para llevar a cabo la estimación de gas metano por el modelo EPA, se tuvo en cuenta la cantidad de residuos dispuestos en el relleno sanitario Doña Juana Zona II área 3 que fueron acumulados en el año 2008 y 2009 con un total de 523.675,584 toneladas; los parámetros de $\mathrm{L}_{0}$ (Índice de Generación de Potencial de Metano) y K (Índices de Generación de Metano), los cuales son dependientes sobre la fracción orgánica disponible, la temperatura y contenido de humedad de los desechos, en donde, se asume que la composición de gas metano es del 50\% aproximadamente. Los valores de $\mathrm{L}_{0} \mathrm{y}$ para $\mathrm{K}$ se pueden observar en la tabla 1.

Tabla 1. Parámetros para Lo y $\mathrm{K}$ para rellenos convencionales, Modelo EPA

\begin{tabular}{cc}
\hline Parámetros del modelo & Valor \\
\hline K & 0.050 por año \\
Lo & $170 \mathrm{~m} 3 /$ ton. \\
\hline Fuente:
\end{tabular}

Fuente: EPA. Landfill Gas Emissions Model. 2006 ${ }^{10}$

\section{Modelo Mexicano}

El modelo asume que el período es de un año desde la colocación de los residuos y el comienzo de la generación de biogás, además que por cada unidad de residuos, después de un año la generación disminuye exponencialmente mientras la fracción orgánica de los residuos es consumida ${ }^{9}$.
La expresión del modelo es la siguiente:

$$
Q_{M_{i=1}^{n}}=\sum 2 k L_{0} M_{i}\left(e^{-k t_{i}}\right) \text {, donde } \sum_{i=1}^{n}=
$$

Suma desde el año de apertura $+1 \quad(\mathrm{i}=1)$ hasta el año de proyección (n); $\mathrm{Q}_{\mathrm{M}}=$ Índice de generación de biogás ( $\mathrm{m}^{3} /$ año); $\mathrm{K}=$ Índice de decaimiento de metano (1/ año); $\mathrm{L}_{0}=$ Potencial máximo de generación de metano $\left(\mathrm{m}^{3} / \mathrm{Mg}\right) ; \mathrm{M}_{\mathrm{I}}=$ Masa de residuos sólidos dispuestos en el año it $; t_{I}=$ Edad de los residuos depositados en año it $^{\text {(años }}{ }^{9}$. El metano generado se calcula usando los índices $\mathrm{L}_{0}$ = Generación Potencial de Metano y k = Índice de Generación de Metano/año, parámetros que el modelo trae incluidos y los cuales se pueden observar en las tablas 2 y 3.

Tabla 2. Índices de Generación Potencial de Metano Lo. Modelo Mexicano

\begin{tabular}{cc}
\hline $\begin{array}{c}\text { Precipitación anual (MM / } \\
\text { AÑO) }\end{array}$ & LO (M 3 /Toneladas) \\
$0-249$ & 60 \\
$250-499$ & 80 \\
$\geq 500$ & 84 \\
\hline
\end{tabular}

Fuente: Stege G. Manual del usuario: modelo Mexicano de biogás. 200911

Tabla 3. Índices de Generación de Metano (K). Modelo Mexicano

\begin{tabular}{cc}
\hline $\begin{array}{c}\text { Precipitación anual } \\
\text { (mm/año) }\end{array}$ & K (por año) \\
\hline $0-249$ & 0.040 \\
$250-499$ & 0.050 \\
$500-999$ & 0.065 \\
$>1000$ & 0.080 \\
\hline
\end{tabular}

Fuente: Stege G. Manual del usuario: modelo Mexicano de biogás. 200911

El total de residuos sólidos utilizados de la zona II área 3 del relleno sanitario Doña Juana, fue de $249.862,913$ toneladas para el año 2008 y para el 2009 un total de 273.812,6717 toneladas. Se asume el $60 \%$ de eficiencia de captura (es la eficiencia con la cual el sistema de captura recuperara el biogás), se utiliza el promedio de la precipitación anual (mm/año). Y un valor de $\mathrm{K}$ (Índice de Generación de Metano): para una precipitación por año de $553.47 \mathrm{~mm}$ que 
corresponde un valor de 0.065 1/año; $\mathrm{L}_{0}$ (Generación Potencial de Metano) para la misma precipitación es de $84 \mathrm{~m}^{3} /$ ton.

\section{Modelo IPCC}

Este modelo se basa en el método de descomposición de primer orden en donde el carbono se degrada formando metano. La expresión matemática de este modelo es la siguiente:

$$
\text { Emisiones de } \mathrm{CH}_{4}=\left[\sum_{x} \mathrm{CH}_{4} \text { generado }_{x, T}-R_{T}\right] \bullet\left(1-\mathrm{OX}_{T}\right)
$$

Donde emisiones de $\mathrm{CH}_{4}$ es la cantidad de metano durante el año T en Gg; T es el año del inventario; $\mathrm{x}$ es la categoría o tipo de desecho y/o material; $\mathrm{R}_{\mathrm{T}}$ es el metano recuperado durante el año; $\mathrm{OX}_{\mathrm{T}}$ factor de oxidación durante el año T. (Grupo Intergubernamental de Expertos Sobre Cambio Climático. 2006). Asimismo, el modelo menciona la entrada de la cantidad de materia orgánica degradable (DOCm), que se encuentra contenida en los residuos eliminados en los sitios de eliminación de desechos sólidos -SEDS-. "El metano potencial que se genera a través de los años se estima sobre la base de las cantidades y la composición de los desechos eliminados en los SEDS y de las prácticas de gestión en los sitios de eliminación. La base para el cálculo es la cantidad de carbono orgánico degradable disuelto -DDOC$" 12$.

Por lo que se tiene que la ecuación $D D O C m=W * D O C * D O C f * M C F$, donde DDOCm es la masa del carbono orgánico degradable disuelto -DDOC- dada en Gg; W es la masa de los desechos depositados; DOC es el carbono orgánico degradable durante el año de deposición: DOCf fracción del DDOC que se puede descomponer; MCF es el factor de corrección de $\mathrm{CH}_{4}$ para la descomposición aeróbica durante el año de descomposición. (IPCC, 2006). De otra parte, se tiene que el potencial de generación de metano $L_{o}=D D O C m \bullet F \bullet 16 / 12$ es igual al producto del DDCOm, la concentración de metano en el gas (F) y el cociente del peso molecular del metano y el C (16/12) ; lo que conlleva a la ecuación, donde Lo es el potencial de generación de metano; DDOCm masa del carbono orgánico degradable -DDOC- disuelto depositado; F fracción del metano en el gas del vertedero generado y $16 / 12$ es el cociente de pesos moleculares $\mathrm{CH}_{4} / \mathrm{C}_{11}$.

La entrada principal de este modelo es la cantidad de materia orgánica degradable (DOCm) contenida en los residuos y los diferentes tipos de este, la cantidad de residuos sólidos ingresados para el año 2008 fue de 249.862,913 toneladas. De otra parte, a este modelo también se ingresaron valores de índice de generación de metano $(\mathrm{K})$, valor que el modelo asume para Bogotá un clima seco tropical con temperaturas mayores a $20^{\circ} \mathrm{C}$ y con precipitaciones menores a 1000 mm/año (ver tabla 4); Carbono orgánico degradable -DOC-, fracción de Carbono orgánico degradable que se descompone (DOCf), el cual tiene un valor por defecto recomendado por el modelo de 0.5 ,valor que se asume de manera hipotética para ambientes anaerobios en los rellenos sanitarios, cuyo tiempo de retardo que el modelo considera un valor por defecto de 6 meses , lo que equivale al tiempo donde se lleva a cabo la reacción para la generación de metano al año siguiente en el cual fueron depositados los residuos sólidos.

Tabla 4. Índice de Generación de metano (K). Modelo IPCC

\begin{tabular}{llrr}
\hline \multicolumn{1}{c}{$\begin{array}{c}\text { Tipo de } \\
\text { desechos }\end{array}$} & \multicolumn{1}{c}{$\begin{array}{c}\text { Índice de generación } \\
\text { de metano (K) }\end{array}$} & Rango & $\begin{array}{c}\text { Valores } \\
\text { defecto }\end{array}$ \\
\hline $\begin{array}{l}\text { Degradación } \\
\text { moderada }\end{array}$ & $\begin{array}{l}\text { Residuos de Alimentos, } \\
\text { jardín }\end{array}$ & $0.05-0.08$ & 0,065 \\
$\begin{array}{l}\text { Degradación } \\
\text { lenta }\end{array}$ & Papel y textil & $0.04-0.06$ & 0,045 \\
Degradación & Madera y paja & $0.02-0.04$ & 0,025 \\
rápida & $\begin{array}{l}\text { Residuos de alimentos y } \\
\text { lodos de aguas hervidas }\end{array}$ & $0,07-0,1$ & 0,085 \\
\hline \multicolumn{3}{c}{ Fuente: Grupo Intergubernamental de Expertos Sobre Cambio } \\
\multicolumn{3}{c}{ Climático. 2006 } \\
Directrices IPCC, 2006 para los inventarios nacionales de gases de \\
\multicolumn{3}{c}{ efecto invernadero. ${ }^{12}$} \\
\end{tabular}

\section{Resultados y discusión}

\section{Modelo EPA}

Una vez ingresada la cantidad de residuos sólidos para este modelo y después de obtener los 
resultados se procedió a hacer el siguiente análisis estadístico:

Tabla 5. Valores de gas metano con el modelo EPA. Relleno sanitario Doña Juana. Proyección 2008-2030

\begin{tabular}{lr}
\hline \multicolumn{2}{c}{ Modelo EPA (m³/año) } \\
\hline Media & $2,14 \mathrm{E}+05$ \\
Desviación estándar & $8,29 \mathrm{E}+04$ \\
Mínimo & $0,00 \mathrm{E}+00$ \\
Máximo & $3,45 \mathrm{E}+05$ \\
Nivel de confianza (95,0\%) & $3,58 \mathrm{E}+04$ \\
\hline
\end{tabular}

El promedio de gas metano generado para los años 2008-2030 corresponde a 2,14E+05 m³/año, con una desviación estándar equivalente a $8,29 \mathrm{E}+04 \mathrm{~m}^{3} / \mathrm{año}$. El valor mínimo de emisiones corresponde a 0 porque para el año 2008 comenzó el funcionamiento de la Zona II área 3 el valor máximo de emisiones durante el periodo de tiempo comprendido entre los años 20082030 equivale a $3.45 \mathrm{E}+05 \mathrm{~m}^{3} /$ año.

Las observaciones estimadas se visualizan en el gráfico 1.

\section{Gráfico 1. Generación de gas metano. Modelo EPA. Relleno Sanitario Doña Juana Zona II Área 3}

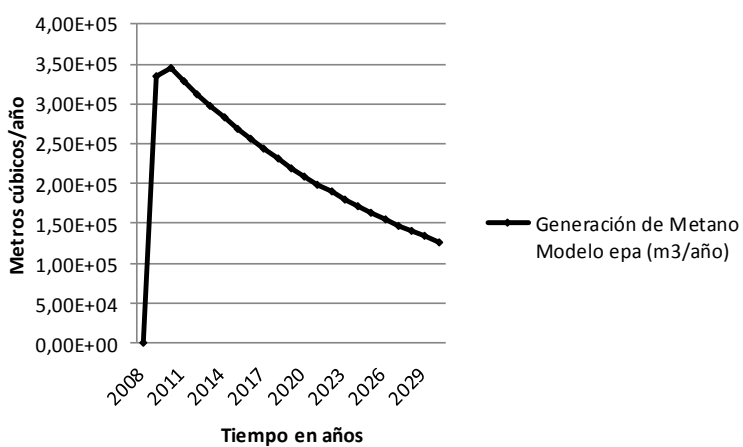

Según el modelo EPA, el pico máximo de producción de metano comienza en el año 2010 con un caudal de $3,45 \mathrm{E}+05 \mathrm{~m}^{3} /$ año, comenzando su fase de decaimiento en el año 2011 para finalmente llegar al año 2030 con un caudal de $1,27 \mathrm{E}+05 \mathrm{~m}^{3} /$ año. Este modelo presenta un decaimiento similar al modelo Mexicano.

\section{Modelo Mexicano}

Una vez ingresada la cantidad de residuos sólidos para este modelo y después de obtener los resultados se procedió a hacer el siguiente análisis estadístico:

Tabla 6. Valores de gas metano con el modelo mexicano

\begin{tabular}{lr}
\hline \multicolumn{2}{c}{ Modelo Mexicano (m³/año) } \\
\hline Media & $2,48 \mathrm{E}+05$ \\
Desviación estándar & $1,16 \mathrm{E}+05$ \\
Mínimo & $0,00 \mathrm{E}+00$ \\
Máximo & $4,46 \mathrm{E}+05$ \\
Nivel de confianza (95,0\%) & $5,02 \mathrm{E}+04$ \\
\hline
\end{tabular}

El promedio de gas metano generado para los años 2008-2030 corresponde a 2,48E+05 m³ /año, con una desviación estándar equivalente a $1,16 \mathrm{E}+05 \mathrm{~m}^{3}$ /año. El valor mínimo de emisiones corresponde a 0 porque para el año 2008 comenzó el funcionamiento de la Zona II área 3 y el valor máximo de emisiones durante el periodo de tiempo comprendido entre los años 20082030 equivale a $4,46 \mathrm{E}+05 \mathrm{~m}^{3} / \mathrm{año}$.

Estas observaciones estimadas se visualizan en el gráfico 2 .

Gráfico 2. Generación de gas metano. Modelo mexicano (m3/año), Relleno Sanitario Doña Juana Zona II Área 3

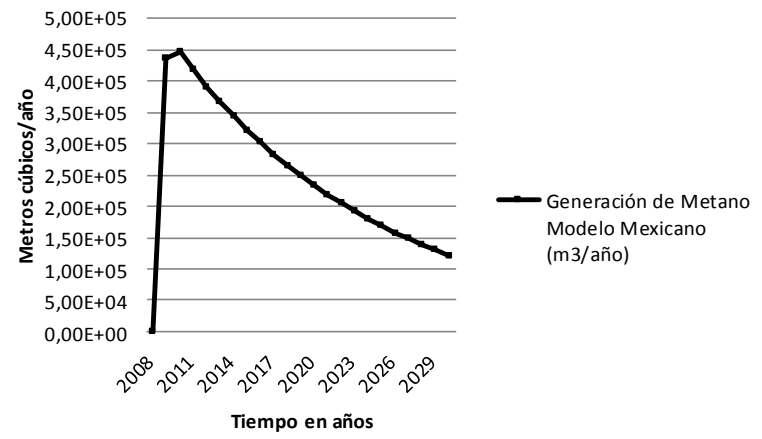

Según el modelo Mexicano, el pico máximo de producción de gas metano comienza en el año 2010 con un caudal de 4,46E+05 m³/año, comenzando su fase de decaimiento en el año 2011 y cuya estimación para el año 2030 es de $1,22 \mathrm{E}+05 \mathrm{~m}^{3}$ /año. 


\section{Modelo IPCC}

Una vez ingresada la cantidad de residuos sólidos para el año 2008 se realizó el siguiente análisis estadístico:

Tabla 7. Valores de gas metano con el Modelo IPCC

\begin{tabular}{lr}
\hline \multicolumn{2}{c}{ Modelo IPCC $\left(\mathbf{m}^{3} / \mathbf{a n ̃ o}\right)$} \\
\hline Media & $6,71 \mathrm{E}+09$ \\
Desviación estándar & $2,71 \mathrm{E}+09$ \\
Mínimo & $0,00 \mathrm{E}+00$ \\
Máximo & $9,37 \mathrm{E}+09$ \\
Nivel de confianza $(95,0 \%)$ & $1,17 \mathrm{E}+09$ \\
\hline
\end{tabular}

El promedio de gas metano generado para los años $2008-2030$ corresponde a $6,71 \mathrm{E}+09 \mathrm{~m}^{3} /$ año, con una desviación estándar equivalente a 2,71E+09 $\mathrm{m}^{3} /$ año. El valor mínimo de emisiones corresponde 0 porque para el año 2008 comenzó el funcionamiento de la Zona II área 3 y el valor máximo de emisiones durante el periodo de tiempo comprendido entre los años 2008-2030 equivale a 9,37E+09 m³/año.

Las observaciones estimadas se visualizan en el gráfico 3.

Gráfico 3. Generación de gas metano. Modelo IPCC. Relleno Sanitario Doña Juana Zona II Área 3

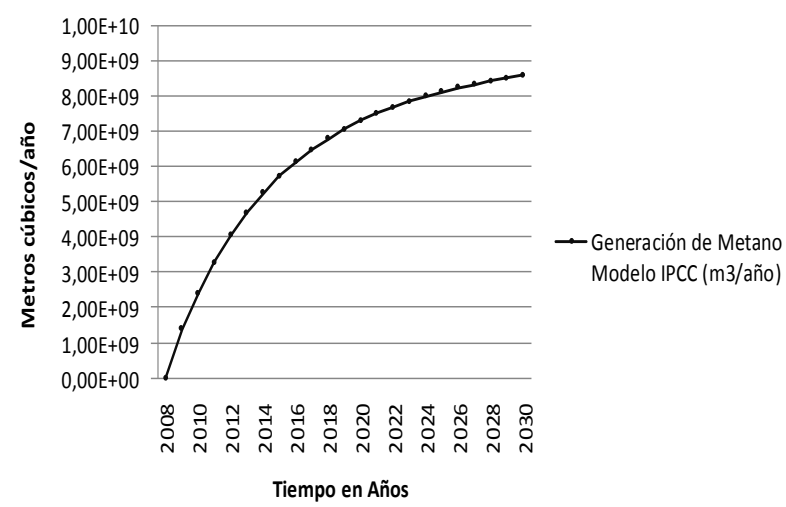

Según el modelo IPCC, la generación de gas metano comienza en el año 2009 con un caudal de $1,41 \mathrm{E}+09 \mathrm{~m}^{3} / \mathrm{año}$, el cual tiene un crecimiento exponencial, llegando al año 2030 con un caudal 9,37E+09 $\mathrm{m}^{3} /$ año, sin observarse decaimiento pues este modelo estima que después de unas cuantas décadas comienza el decaimiento del gas metano, para nuestro caso el decaimiento empieza a partir de la segunda década es decir para el año 2031.

Es de anotar, que los tres modelos, son de tipo exponencial, lo que quiere decir que modelan el crecimiento de una determinada población durante un período de tiempo, para luego mostrar un decaimiento el cual es debido a que la producción de gas metano se hace más lenta en comparación a los dos primeros años y luego considera un disminución exponencial en el tiempo de predicción. Las metodologías de la EPA y mexicano, en su configuración de las familias de ecuaciones que lo construyen, presentan similitudes en el análisis del fenómeno a predecir, sin embargo las constantes de cada modelo y los datos de ingreso, varían en la cualificación y desde luego en la predicción del biogás en el relleno sanitario.

En Latinoamérica, usualmente la metodología de la EPA y Mexicana, están siendo utilizadas en la predicción del biogás en rellenos sanitarios, en condiciones y características de otros países como Estados Unidos y México, en donde la composición de los residuos y climatología son muy diferentes a la de Colombia ${ }^{13}$; sin embargo, la metodología del IPCC por las condiciones de la configuración de las familias de ecuaciones que lo componen así como las constantes que se asumen para la predicción y los datos de ingresos, generan una amplia distorsión en los datos estimados de biogás, lo cual, generan una desviación cuantitativa de los datos en una toma de decisiones, para el aprovechamiento del biogás, dado que puede ser un dato incrementado que no corresponde con la realidad de la producción de biogás en el relleno sanitario.

En la tabla 8, se observa una comparación de los resultados con las diferentes metodologías aplicadas en términos nominales de la producción de biogás y el análisis estadístico, el cual es una herramienta para la toma de decisiones en la estimación óptima del biogás en el Relleno Sanitario estudiado. 
Tabla 8. Comparación de valores de biogás con la aplicación de los tres modelos

\begin{tabular}{lccc}
\hline \multicolumn{1}{c}{$\begin{array}{c}\text { Aspecto a } \\
\text { evaluar }\end{array}$} & $\begin{array}{c}\text { Modelo } \\
\text { mexicano }\end{array}$ & $\begin{array}{c}\text { Modelo } \\
\text { EPA }\end{array}$ & $\begin{array}{c}\text { Modelo } \\
\text { IPCC }\end{array}$ \\
\hline $\begin{array}{l}\text { Promedio } \\
\text { biogas }\left(\mathrm{m}^{3} / \mathrm{año}\right)\end{array}$ & $2.48 \mathrm{E}+05$ & $2.14 \mathrm{E}+05$ & $6.71 \mathrm{E}+05$ \\
$\begin{array}{l}\text { Desviación } \\
\text { estandar } \\
\left(\mathrm{m}^{3} / \text { año }\right)\end{array}$ & $1.16 \mathrm{E}+05$ & $8.29 \mathrm{E}+05$ & $2.71 \mathrm{E}+05$ \\
$\begin{array}{l}\text { Error cuadrático } \\
\text { medio }\end{array}$ & $4.28 \mathrm{E}+05$ & $7.87 \mathrm{E}+05$ & $8.21 \mathrm{E}+05$ \\
\hline
\end{tabular}

\section{Conclusiones}

Es significativo señalar, que al comparar los métodos mexicano, EPA e IPCC, en la aplicación de los mismos para la estimación del biogás en el relleno sanitario, estos, predicen la cantidad de biogás, simulando una degradación exponencial, dado que aunque no se conocen las reacciones químicas que describen el proceso de digestión anaerobia en el relleno sanitario en cuanto al biogás, la estimación con las metodologías antes descritas, son una buena aproximación para conocer el fenómeno de estudio.

Al utilizar el modelo IPCC, presenta un comportamiento atípico en comparación a los otros dos modelos (EPA y mexicano), aunque tiene un crecimiento exponencial, presenta en su fase de decaimiento, después de unas cuantas décadas de iniciada la producción de gas metano, para nuestro caso es en la segunda década es decir aproximadamente para el año 2031, para este caso, es de aproximadamente 20 años, después de cumplidas dos décadas. Al aplicar los modelos de la EPA y mexicano, se presenta una similitud en sus ecuaciones de estimación y un mejor ajuste a la expresión del fenómeno estudiado, aunque la calibración de estos modelos es fundamental, para correlacionar los datos obtenidos y medidos; sin embargo el mexicano, es el método que presenta mejor resultado de estimación promedio de biogás y ajuste estadístico adecuado en este caso de estudio.

Conflicto de intereses: Ninguno declarado por autores.

\section{Referencias}

1. Mebark-Adouane B, Khaldi-Dehimi S, Haddad D. Theoretical estimation of the production of biogas from the landfill of batna city and its electrical conversion by a sofc. Renewable and sustainable energy reviews. 2015;41:277-283.

2. Nadaletti W, Cremonez P, De Souza S, Bariccatti R, Belli P. 2015. Potential use of landfill biogas in urban bus fleet in the brazilian states: a review. Renewable and sustainable energy reviews. 2015;41:277-283.

3. Hincapié I, Estévez S, Giraldo E. Análisis y comportamiento de compuestos orgánicos volátiles en las emisiones de biogás del proveniente del relleno sanitario de doña juana. Universidad de los andes. 2001:1-4.

4. Figueroa V, Cooper D, Mackie K. Estimating landfill greenhouse gas emissions from measured ambient methane concentrations and dispersion modeling. 2008;327 (1): 1-17.

5. Manosalva MP. Estimación del flujo superficial de metano (ch4) en las emisiones del biogás desde la zona vii del relleno sanitario de doña juana. 2005.

6. Meraz, R, Dominguez A. Estudio experimental a escala piloto y modelización de la producción de biogás en relleno sanitario. 2000. Disponible en http://www.bvsde.paho.org/bvsacd/ciiemad/estudio. pdf

7. Tchobanoglous G, Theisen H, Vigil. Gestión integral de residuos sólidos. Volumen l. Madrid: mc graw hill interamericana de españa. 1994.

8. Bryman A. Social research methods. Second edition. New york: oxford university press. 2003.

9. Usepa. Modelo centro americano de biogás. Program landfill methane outreach (u.s.Epa), Washington, DC. $2007 . \quad$ Disponible en http://www.epa.gov/lmop/documents/pdfs/manuald elusuariov1_centro

10. Epa. Landfill gas emissions model. Washington, DC. $2006 . \quad$ Disponible en http://www.epa.gov/ttncatc1/dir1/landgem-v302guide.pdf

11. Estege G, Dávila J. Manual de usuario, modelo mexicano de biogás. Landfill methane. Agencia para la protección del ambiente (u.s.Epa). 2009: 25.

12. Grupo Intergubernamental de expertos sobre cambio climático. Directrices IPCC, 2006 para los inventarios nacionales de gases de efecto invernadero. Modulo desechos. 2006. Disponible en http://www.ipccnggip.iges.or.jp/public/gl/spanish.html

13. Ludwig V. 2008. Perspectiva general de la asociación metano a los mercados - sector rellenos sanitarios. Methane to markets. Disponible en http://epa.gov/gasstar/documents/workshops/bueno saires-2008/m2m_overview_sp.pdf 